\title{
Versatile and multivalent nanobodies efficiently neutralize SARS-CoV-2
}

\author{
Yufei Xiang', Sham Nambulli ${ }^{2,3 *}$, Zhengyun Xiao ${ }^{1 *}$, Heng Liu ${ }^{4 *}$, Zhe Sangi,5, W. Paul Duprex ${ }^{2,3}$, \\ Dina Schneidman-Duhovny ${ }^{6}+$, Cheng Zhang ${ }^{4}+$, Yi Shi ${ }^{1,5} \dagger$
}

${ }^{1}$ Department of Cell Biology, University of Pittsburgh, Pittsburgh, PA, USA. ${ }^{2}$ Center for Vaccine Research, University of Pittsburgh, Pittsburgh, PA, USA. ${ }^{3}$ Department of Microbiology and Molecular Genetics, University of Pittsburgh, Pittsburgh, PA, USA. ${ }^{\circ}$ Department of Pharmacology and Chemical Biology, University of Pittsburgh, Pittsburgh, PA, USA. 5Pitt/CMU Program for Computational Biology, Pittsburgh, PA, USA. ${ }^{6}$ School of Computer Science and Engineering, Institute of Life Sciences, The Hebrew University of Jerusalem, Israel.

*These authors contributed equally to this work.

†Corresponding author. Email: dina.schneidman@mail.huji.ac.il (D.S.-D.); chengzh@pitt.edu (C.Z.); yi.shi@pitt.edu (Y.S.)

Cost-effective, efficacious therapeutics are urgently needed against the COVID-19 pandemic. Here, we used camelid immunization and proteomics to identify a large repertoire of highly potent neutralizing nanobodies (Nbs) to the SARS-CoV-2 spike (S) protein receptor-binding domain (RBD). We discovered Nbs with picomolar to femtomolar affinities that inhibit viral infection at sub-ng/ml concentration and determined a structure of one of the most potent in complex with RBD. Structural proteomics and integrative modeling revealed multiple distinct and non-overlapping epitopes and indicated an array of potential neutralization mechanisms. We constructed multivalent $\mathrm{Nb}$ constructs that achieved ultrahigh neutralization potency (IC50s as low as $0.058 \mathrm{ng} / \mathrm{ml}$ ) and may prevent mutational escape. These thermostable Nbs can be rapidly produced in bulk from microbes and resist lyophilization, and aerosolization.

Globally a novel, highly transmissible coronavirus severe acute respiratory syndrome coronavirus 2 (SARS-CoV-2) $(1,2)$ has infected more than 30 million people and has claimed almost 1,000,000 lives, with the numbers still rising as of September 2020. Despite preventive measures, such as quarantines and lock-downs that help curb viral transmission, the virus rebounds after lifting social restrictions. Safe and effective therapeutics and vaccines remain in dire need.

Like other zoonotic coronaviruses, SARS-CoV-2 expresses a surface spike (S) glycoprotein, which consists of S1 and S2 subunits forming a homotrimeric viral spike to interact with host cells. The interaction is mediated by the S1 receptor-binding domain (RBD), which binds the peptidase domain (PD) of angiotensin-converting enzyme-2 (hACE2) as a host receptor (3). Structural studies have revealed different conformations of the spike $(4,5)$. In the pre-fusion stage, the RBD switches between a closed conformation, and an open conformation for hACE2 interaction. In the post-fusion stage, the S2 undergoes a dramatic conformational change to trigger host membrane fusion (6). Investigations into COVID-19 convalescence individuals' sera have led to the identification of potent neutralizing antibodies (NAbs) primarily targeting the RBD but also non-RBD epitopes (7-13). High-quality NAbs may overcome the risks of $\mathrm{Fc}$-associated antibody-dependent enhancement (ADE) and are promising therapeutic candidates $(14,15)$.
$\mathrm{V}_{\mathrm{H}} \mathrm{H}$ antibodies or nanobodies (Nbs) are minimal, monomeric antigen-binding domains derived from camelid singlechain antibodies (16). Unlike IgG antibodies, Nbs are small $(\sim 15 \mathrm{kDa})$, highly soluble and stable, readily bioengineered into bi/multivalent forms, and are amenable to low-cost, efficient microbial production. Due to their robust physicochemical properties, Nbs can be administered by inhalation, making their use against the respiratory viruses very appealing $(17,18)$. Recently, several SARS-CoV-2 neutralizing Nbs have been identified, by screening SARS-CoV or Middle East respiratory syndrome (MERS) cross-reacting Nbs or using synthetic Nb libraries for RBD binding. However, these synthetic Nbs generally neutralize the virus at $\mu \mathrm{g}$ to sub $-\mu \mathrm{g} / \mathrm{ml}$ concentration $(12,19-22)$, which are hundreds of times less potent than the best NAbs, likely due to monovalency and lack of affinity maturation $(23,24)$. The development of highly potent anti-SARS-CoV-2 Nbs may provide a novel means for versatile, cost-effective therapeutics and point-ofcare diagnosis.

To produce high-quality SARS-CoV-2 neutralizing Nbs, we immunized a llama with the recombinant RBD. Compared to the pre-bleed, the post-immunized serum showed potent and specific serologic activities toward RBD binding with a titer of $1.75 \times 10^{6}$ (fig. S1A). The serum efficiently neutralized the pseudotyped SARS-CoV-2 at the half-maximal neutralization titer (NT50) of $\sim 310,000$ (fig. S1B), orders of magnitude 
higher than the convalescent sera obtained from recovered COVID-19 patients $(7,8)$. To further characterize these activities, we separated the single-chain $\mathrm{V}_{\mathrm{H}} \mathrm{H}$ antibodies from the IgGs. We confirmed that the single-chain antibodies achieve specific, high-affinity binding to the RBD and possess sub-nM half-maximal inhibitory concentration $($ IC50 $=509 \mathrm{pM}$ ) against the pseudotyped virus (fig. S1C).

We identified thousands of high-affinity $\mathrm{V}_{\mathrm{H}} \mathrm{H}$ Nbs from the RBD-immunized llama serum using a robust proteomic strategy that we have recently developed (25) (fig. S2A). This repertoire includes $\sim 350$ unique CDR3s (complementaritydetermining regions). For E. coli expression, we selected 109 highly diverse $\mathrm{Nb}$ sequences from the repertoire with unique CDR3s to cover various biophysical, structural, and potentially different antiviral properties. $94 \mathrm{Nbs}$ were purified and tested for RBD binding by ELISA, from which we confirmed 71 RBD-specific binders (fig. S2, B and C, and tables S1 and S4). Of these RBD-specific binders, $49 \mathrm{Nbs}$ presented high solubility and high-affinity (ELISA IC50 below $30 \mathrm{nM}$, Fig. $1 \mathrm{~A}$ ), and were promising candidates for functional characterizations. We used a SARS-CoV-2-GFP pseudovirus neutralization assay to screen and characterize the antiviral activities of these high-affinity Nbs. $94 \%$ of the tested Nbs neutralize the pseudotype virus below $3 \mu \mathrm{M}$ (Fig. 1B), with $90 \%$ of them below $500 \mathrm{nM}$. Only $20-40 \%$ of high-affinity RBD-specific mAbs identified from patient sera have been reported to possess comparable potency $(7,8)$. Over three quarters $(76 \%)$ of the Nbs efficiently neutralized the pseudovirus below $50 \mathrm{nM}$, and $6 \%$ had neutralization activities below $0.5 \mathrm{nM}$. We selected the most potent 18 based on the pseudovirus GFP reporter screen and measured their potency accurately using the pseudovirus-luciferase reporter assay. Finally, we tested the potential of 14 to neutralize the SARS-CoV-2 Munich strain using the PRNT50 assay (26). All the Nbs reached 100\% neutralization and neutralized the virus in a dose-dependent manner. The IC50s span from single-digit $\mathrm{ng} / \mathrm{ml}$ to sub$\mathrm{ng} / \mathrm{ml}$, of the 3 most potent $\mathrm{Nbs} 89,20$, and 21 , showed neutralization of $2.1 \mathrm{ng} / \mathrm{ml}(0.133 \mathrm{nM}), 1.6 \mathrm{ng} / \mathrm{ml}(0.102 \mathrm{nM})$, and $0.7 \mathrm{ng} / \mathrm{ml}(0.045 \mathrm{nM})$, respectively, in the pseudovirus assay (Fig. 1C) and $0.154 \mathrm{nM}, 0.048 \mathrm{nM}$, and $0.022 \mathrm{nM}$, in the SARSCoV-2 assay (Fig. 1, D and E). Overall, there was an excellent correlation between the two neutralization assays $\left(R^{2}=0.92\right.$, fig. S3).

We measured the binding kinetics of $\mathrm{Nbs} 89,20$, and 21 by surface plasmon resonance (SPR) (fig. S4, A and B). Nbs 89 and 20 have an affinity of $108 \mathrm{pM}$ and $10.4 \mathrm{pM}$, and the most potent $\mathrm{Nb} 21$ did not show detectable dissociation from the RBD during 20 min SPR analysis. The sub-picomolar affinity of $\mathrm{Nb} 21$ potentially explains its unusual neutralization potency (Fig. 1F). We determined the thermostability of Nbs 89, 20, and 21 from the E. coli periplasmic preparations to be 65.9 , 71.8, and $72.8^{\circ} \mathrm{C}$, respectively (fig. S4C). Finally, we tested the on-shelf stability of $\mathrm{Nb21}$, which remained soluble after $\sim 6$ weeks of storage at room temperature after purification. No multimeric forms or aggregations were detected by size-exclusion chromatography (SEC) (fig. S4D). Together these results suggest that these neutralizing Nbs have excellent physicochemical properties for advanced therapeutic applications.

We employed an integrative approach by SEC, cross-linking/ mass spectrometry, and structural modeling for epitope mapping. (27-30). First, we performed SEC experiments to distinguish between $\mathrm{Nbs}$ that share the same RBD epitope as $\mathrm{Nb} 21$ and those that bind to non-overlapping epitopes. Nbs 9, 16, $17,20,64,82,89,99$, and 107 competed with Nb21 for RBD binding based on SEC profiles (Fig. 2A and fig. S5), indicating that their epitopes significantly overlap. In contrast, higher mass species (from early elution volumes) corresponding to the trimeric complexes composed of $\mathrm{Nb21}, \mathrm{RBD}$, and one of the $\mathrm{Nbs}(34,36,93,105$, and 95) were evident (Fig. 2B and fig. $\mathrm{S} 6, \mathrm{~A}$ to $\mathrm{H}$ ). Moreover, Nb105 competed with $\mathrm{Nb} 34$ and $\mathrm{Nb} 95$, which did not compete for RBD interaction, suggesting the presence of two distinct and non-overlapping epitopes. Second, we cross-linked Nb-RBD complexes by DSS (disuccinimidyl suberate) and identified on average, four intermolecular cross-links by MS for Nbs 20, 93, 34, 95, and 105. The cross-links were used to map the RBD epitopes derived from the SEC data (Methods). Our cross-linking models identified five epitopes (I, II, III, IV, and V corresponding to Nbs $20,93,34,95$, and 105) (Fig. 2C). The models satisfied $90 \%$ of the cross-links with an average precision of $7.8 \AA$ (Fig. 2D and table S2). Our analysis confirmed the presence of a dominant Epitope I (e.g., epitopes of Nbs 20 and 21) overlapping with the hACE2 binding site. Epitope II also co-localized with the non-conserved hACE2 binding site. Both epitopes I and II Nbs can compete with hACE2 binding to RBD at very low concentrations in vitro (fig. S7A). Epitopes III-V colocalized with conserved sites (fig. S7, B and C). Interestingly, epitope I Nbs had significantly shorter CDR3 (four amino acids shorter, $p=0.005$ ) than other epitope binders (fig. S6I). Despite this, the vast majority of the selected Nbs potently inhibited the virus with an IC50 below $30 \mathrm{ng} / \mathrm{ml}(2 \mathrm{nM})$ (table S1).

To explore the molecular mechanisms that underlie the potent neutralization activities of Epitope I Nbs, we determined a crystal structure of the RBD-Nb20 complex at a resolution of $3.3 \AA$ by molecular replacement (Methods, table S3, and fig. S13). Most of the residues in RBD (N334-G526) and the entire $\mathrm{Nb20}$, particularly those at the protein interaction interface, are well resolved. There are two copies of RBD$\mathrm{Nb} 20$ complexes in one asymmetric unit, which are almost identical with an RMSD of $0.277 \AA$ over $287 \mathrm{C} \alpha$ atoms. In the structure, all three CDRs of $\mathrm{Nb} 20$ interact with the RBD by binding to its large extended external loop with two short 
$\beta$-strands (Fig. 3A) (31). E484 of RBD forms hydrogen bonding and ionic interactions with the side chains of R31 (CDR1) and Y104 (CDR3) of Nb20, while Q493 of RBD forms hydrogen bonds with the main chain carbonyl of A29 (CDR1) and the side chain of R97 (CDR3) of Nb20. These interactions constitute a major polar interaction network at the RBD and $\mathrm{Nb} 20$ interface. $\mathrm{R} 31$ of $\mathrm{Nb} 20$ also engages in a cation- $\pi$ interaction with the side chain of F490 of the RBD (Fig. 3B). In addition, M55 from the $\mathrm{CDR} 2$ of $\mathrm{Nb} 20$ packs against residues L452, F490, and L492 of RBD to form hydrophobic interactions at the interface. Another small patch of hydrophobic interactions is formed among residues V483 of RBD and F45 and L59 from the framework $\beta$-sheet of Nb20 (Fig. 3C).

The binding mode of $\mathrm{Nb} 20$ to $\mathrm{RBD}$ is distinct from other reported neutralizing $\mathrm{Nbs}$, which generally recognize similar epitopes in the RBD external loop region (32-34) (fig. S8). The extensive hydrophobic and polar interactions (Fig. 3, B and $\mathrm{C}$ ) between $\mathrm{RBD}$ and $\mathrm{Nb20}$ stem from the remarkable shape complementarity (Fig. 3D) between the CDRs and the external RBD loop, leading to ultrahigh-affinity ( 10 pM). We further modeled the structure of the best neutralizer $\mathrm{Nb} 21$ with RBD based on our crystal structure (Methods). Only four residues vary between $\mathrm{Nb} 20$ and $\mathrm{Nb} 21$ (fig. S9A), all of which are on CDRs. Two substitutions are at the RBD binding interface. S52 and M55 in the CDR2 of Nb20 are replaced by two asparagine residues $\mathrm{N} 52$ and $\mathrm{N} 55$ in Nb21. In our superimposed structure, N52 forms a new H-bond with N450 of RBD (fig. S9B). While N55 does not engage in additional interactions with RBD, it creates a salt bridge with the side chain of R31, which stabilizes the polar interaction network among R31 and Y104 of Nb21 and Q484 of RBD (fig. S9B). All of those likely contribute to a slower off-rate of $\mathrm{Nb} 21$ (Fig. $1 \mathrm{~F}$ and fig. S4A) and stronger neutralization potency. Structural comparison of RBD-Nb20/21 and RBD-hACE2 (PDB 6LZG) (31) clearly showed that the interfaces for $\mathrm{Nb20} / 21$ and hACE2 partially overlap (Fig. 3D and fig. S9C). Notably, the CDR1 and CDR3 of $\mathrm{Nb20} / 21$ would clash with the first helix of hACE2, the primary binding site for RBD (fig. S9D).

To understand the antiviral efficacy of our Nbs, we superimposed RBD-Nb complexes to different spike conformations based on cryoEM structures. We found that three copies of $\mathrm{Nb20/21}$ can simultaneously bind all three RBDs in their "down" conformations (PDB 6VXX) (4) that correspond to the inactive spike (Fig. 4B). Our analysis indicates a potential mechanism by which Nbs 20 and 21 (Epitope I) lock RBDs in their down conformation with ultrahigh affinity. Combined with the steric interference with hACE2 binding in the RBD open conformation (Fig. 4A), these mechanisms may explain the exceptional neutralization potencies of Epitope I Nbs.

Other epitope-binders do not fit into this inactive conformation without steric clashes and appear to use different neutralization strategies (Fig. 4C). For example, Epitope II:
$\mathrm{Nb} 93$ co-localizes with the hACE2 binding site and can bind the spike in the one RBD "up" conformation (Fig. 4D, PDB $6 \mathrm{VSB}$ ) (3). It may neutralize the virus by blocking the hACE2 binding site. Epitope III and IV Nbs can only bind when two or three RBDs are in their "up" conformations (PDB 6XCN) (24) where the epitopes are exposed. In the all RBDs "up" conformation, three copies of Nbs can directly interact with the trimeric spike. Interestingly, through RBD binding, Epitope III: Nb34 can be accommodated on top of the trimer to lock the helices of S2 in the prefusion stage, preventing their large conformational changes for membrane fusion (Fig. 4E). When superimposed onto the all "up" conformation, Epitope IV: Nb95 is proximal to the rigid NTD of the trimer, presumably restricting the flexibility of the spike domains (Fig. 4F).

Epitope mapping enabled us to bioengineer homo- and hetero-dimeric and homo-trimeric Nbs. Homodimers/trimers based on $\mathrm{Nb} 20$ or $\mathrm{Nb} 21$ were designed to increase the antiviral activities through avidity binding to the trimeric spike. Heterodimers pairing $\mathrm{Nb} 21$ with $\mathrm{Nbs}$ that bind a different epitope were designed to prevent viral escape. The homodimers/trimers used flexible linker sequences of 25 (GS) or 31 (EK) amino acids (Methods). The heterodimers used flexible linkers of 12 amino acids.

We found up to $\sim 30$ fold improvement for the homotrimeric constructs of $\mathrm{Nb}_{2} 1_{3}(\mathrm{IC} 50=1.3 \mathrm{pM})$ and $\mathrm{Nb}_{2} \mathrm{O}_{3}(\mathrm{IC} 50=$ $4.1 \mathrm{pM}$ ) compared to the respective monomeric form by the pseudovirus luciferase assay (Figs. 1, C and E, and 5, A and C). Similar results were obtained from the SARS-CoV-2 PRNT (Fig. 5, B and C, and fig. S11A). The improvements are likely greater than these values indicate, as the measured values may reflect the assay's lower detection limits. For the heterodimeric constructs, up to a 4-fold increase of potency (i.e., Nb21-Nb34) was observed. The multivalent constructs retained similar physicochemical properties to the monomeric Nbs, including high solubility, yield, thermostability, and remained intact (non-proteolyzed) under the neutralization assay condition (fig. S10). They remained highly potent for pseudovirus neutralization after lyophilization and aerosolization (Methods and fig. S11, B to G), indicating the outstanding stability and potential flexibility of administration. The majority of the RBD mutations observed in GISAID (35) are very low in frequency $(<0.0025)$ which may increase under $\mathrm{Nb}$ selection. Therefore, a cocktail consisting of ultrapotent, multivalent constructs that bind simultaneously a variety of epitopes with potentially different neutralization mechanisms will likely efficiently block virus mutational escape (Fig. 5E and fig. S12) (9, 36-38).

Here, in vivo antibody affinity maturation followed by advanced proteomics (25) enabled the rapid discovery of a diverse repertoire of high-affinity $\mathrm{RBD} \mathrm{Nbs}$, including an ultrapotent neutralizer with sub-picomolar affinity, which is 
unprecedented for natural, single-domain antibodies. We demonstrated the simplicity and versatility of $\mathrm{Nb}$ bioengineering and the outstanding physicochemical properties of the monomeric Nbs and their multivalent forms. To our knowledge, the multivalent constructs represent the most potent SARS-CoV-2 neutralizers to date. Flexible and efficient administration, such as inhalation may further improve their antiviral efficacy while minimizing the dose, cost, and potential toxicity for clinical applications. The high sequence similarity between $\mathrm{Nbs}$ and human IgGs may restrain the immunogenicity (39). It is possible to fuse the antiviral Nbs with highly stable, albumin- $\mathrm{Nb}$ constructs (40) to improve pharmacokinetics. These high-quality Nbs can also be applied as rapid and economic point-of-care diagnostics. We envision that the $\mathrm{Nb}$ technology described here will contribute to curbing the current pandemic and possibly a future event.

\section{REFERENCES AND NOTES}

1. N. Zhu, D. Zhang, W. Wang, X. Li, B. Yang, J. Song, X. Zhao, B. Huang, W. Shi, R. Lu, P. Niu, F. Zhan, X. Ma, D. Wang, W. Xu, G. Wu, G. F. Gao, W. Tan; China Novel Coronavirus Investigating and Research Team, A Novel Coronavirus from Patients with Pneumonia in China, 2019. N. Engl. J. Med. 382, 727-733 (2020). doi:10.1056/NEJMoa2001017 Medline

2. P. Zhou, X.-L. Yang, X.-G. Wang, B. Hu, L. Zhang, W. Zhang, H.-R. Si, Y. Zhu, B. Li, C.L. Huang, H.-D. Chen, J. Chen, Y. Luo, H. Guo, R.-D. Jiang, M.-Q. Liu, Y. Chen, X.-R. Shen, X. Wang, X.-S. Zheng, K. Zhao, Q.-J. Chen, F. Deng, L.-L. Liu, B. Yan, F.-X. Zhan, Y.-Y. Wang, G.-F. Xiao, Z.-L. Shi, A pneumonia outbreak associated with a new coronavirus of probable bat origin. Nature 579, 270-273 (2020). doi:10.1038/s41586-020-2012-7 Medline

3. D. Wrapp, N. Wang, K. S. Corbett, J. A. Goldsmith, C.-L. Hsieh, O. Abiona, B. S. Graham, J. S. McLellan, Cryo-EM structure of the 2019-nCoV spike in the prefusion conformation. Science 367, 1260-1263 (2020). doi:10.1126/science.abb2507 Medline

4. A. C. Walls, Y.-J. Park, M. A. Tortorici, A. Wall, A. T. McGuire, D. Veesler, Structure, Function, and Antigenicity of the SARS-CoV-2 Spike Glycoprotein. Cell 181, 281292.e6 (2020). doi:10.1016/i.cell.2020.02.058 Medline

5. Y. Cai, J. Zhang, T. Xiao, H. Peng, S. M. Sterling, R. M. Walsh Jr., S. Rawson, S. RitsVolloch, B. Chen, Distinct conformational states of SARS-CoV-2 spike protein. Science 369, 1586-1592 (2020). doi:10.1126/science.abd4251 Medline

6. X. Fan, D. Cao, L. Kong, X. Zhang, Cryo-EM analysis of the post-fusion structure of the SARS-CoV spike glycoprotein. Nat. Commun. 11, 3618 (2020). doi:10.1038/s41467-020-17371-6 Medline

7. Y. Cao, B. Su, X. Guo, W. Sun, Y. Deng, L. Bao, Q. Zhu, X. Zhang, Y. Zheng, C. Geng, X. Chai, R. He, X. Li, Q. Lv, H. Zhu, W. Deng, Y. Xu, Y. Wang, L. Qiao, Y. Tan, L. Song, G. Wang, X. Du, N. Gao, J. Liu, J. Xiao, X. D. Su, Z. Du, Y. Feng, C. Qin, C. Qin, R. Jin, X. S. Xie, Potent Neutralizing Antibodies against SARS-CoV-2 Identified by HighThroughput Single-Cell Sequencing of Convalescent Patients' B Cells. Cell 182. 73-84.e16 (2020). doi:10.1016/i.cell.2020.05.025 Medline

8. D. F. Robbiani, C. Gaebler, F. Muecksch, J. C. C. Lorenzi, Z. Wang, A. Cho, M. Agudelo, C. O. Barnes, A. Gazumyan, S. Finkin, T. Hägglöf, T. Y. Oliveira, C. Viant, A. Hurley, H.-H. Hoffmann, K. G. Millard, R. G. Kost, M. Cipolla, K. Gordon, F. Bianchini, S. T. Chen, V. Ramos, R. Patel, J. Dizon, I. Shimeliovich, P. Mendoza, H. Hartweger, L. Nogueira, M. Pack, J. Horowitz, F. Schmidt, Y. Weisblum, E. Michailidis, A. W. Ashbrook, E. Waltari, J. E. Pak, K. E. Huey-Tubman, N. Koranda, P. R. Hoffman, A. P. West Jr., C. M. Rice, T. Hatziioannou, P. J. Bjorkman, P. D. Bieniasz, M. Caskey, M. C. Nussenzweig, Convergent antibody responses to SARS-CoV-2 in convalescent individuals. Nature 584, 437-442 (2020). doi:10.1038/s41586-020-2456-9 Medline

9. J. Hansen, A. Baum, K. E. Pascal, V. Russo, S. Giordano, E. Wloga, B. O. Fulton, Y. Yan, K. Koon, K. Patel, K. M. Chung, A. Hermann, E. Ullman, J. Cruz, A. Rafique, T. Huang, J. Fairhurst, C. Libertiny, M. Malbec, W. Y. Lee, R. Welsh, G. Farr, S. Pennington, D. Deshpande, J. Cheng, A. Watty, P. Bouffard, R. Babb, N.
Levenkova, C. Chen, B. Zhang, A. Romero Hernandez, K. Saotome, Y. Zhou, M. Franklin, S. Sivapalasingam, D. C. Lye, S. Weston, J. Logue, R. Haupt, M. Frieman, G. Chen, W. Olson, A. J. Murphy, N. Stahl, G. D. Yancopoulos, C. A. Kyratsous, Studies in humanized mice and convalescent humans yield a SARS-CoV-2 antibody cocktail. Science 369, 1010-1014 (2020). doi:10.1126/science.abd0827 Medline

10. L. Liu, P. Wang, M. S. Nair, J. Yu, M. Rapp, Q. Wang, Y. Luo, J. F.-W. Chan, V. Sahi, A. Figueroa, X. V. Guo, G. Cerutti, J. Bimela, J. Gorman, T. Zhou, Z. Chen, K.-Y. Yuen, P. D. Kwong, J. G. Sodroski, M. T. Yin, Z. Sheng, Y. Huang, L. Shapiro, D. D. $\mathrm{Ho}$, Potent neutralizing antibodies against multiple epitopes on SARS-CoV-2 spike. Nature 584, 450-456 (2020). doi:10.1038/s41586-020-2571-7 Medline

11. P. J. M. Brouwer, T. G. Caniels, K. van der Straten, J. L. Snitselaar, Y. Aldon, S. Bangaru, J. L. Torres, N. M. A. Okba, M. Claireaux, G. Kerster, A. E. H. Bentlage, M. M. van Haaren, D. Guerra, J. A. Burger, E. E. Schermer, K. D. Verheul, N. van der Velde, A. van der Kooi, J. van Schooten, M. J. van Breemen, T. P. L. Bijl, K. Sliepen, A. Aartse, R. Derking, I. Bontjer, N. A. Kootstra, W. J. Wiersinga, G. Vidarsson, B. L. Haagmans, A. B. Ward, G. J. de Bree, R. W. Sanders, M. J. van Gils, Potent neutralizing antibodies from COVID-19 patients define multiple targets of vulnerability. Science 369, 643-650 (2020). doi:10.1126/science.abc5902 Medline

12. D. Wrapp, D. De Vlieger, K. S. Corbett, G. M. Torres, N. Wang, W. Van Breedam, K. Roose, L. van Schie, M. Hoffmann, S. Pöhlmann, B. S. Graham, N. Callewaert, B. Schepens, X. Saelens, J. S. McLellan; VIB-CMB COVID-19 Response Team, Structural Basis for Potent Neutralization of Betacoronaviruses by Single-Domain Camelid Antibodies. Cell 181, 1436-1441 (2020). doi:10.1016/i.cell.2020.05.047 Medline

13. A. Z. Wec, D. Wrapp, A. S. Herbert, D. P. Maurer, D. Haslwanter, M. Sakharkar, R. K. Jangra, M. E. Dieterle, A. Lilov, D. Huang, L. V. Tse, N. V. Johnson, C.-L. Hsieh, N. Wang, J. H. Nett, E. Champney, I. Burnina, M. Brown, S. Lin, M. Sinclair, C. Johnson, S. Pudi, R. Bortz 3rd, A. S. Wirchnianski, E. Laudermilch, C. Florez, J. M. Fels, C. M. O'Brien, B. S. Graham, D. Nemazee, D. R. Burton, R. S. Baric, J. E. Voss, K. Chandran, J. M. Dye, J. S. McLellan, L. M. Walker, Broad neutralization of SARSrelated viruses by human monoclonal antibodies. Science 369, 731-736 (2020). doi:10.1126/science.abc7424 Medline

14. T. Zohar, G. Alter, Dissecting antibody-mediated protection against SARS-CoV-2. Nat. Rev. Immunol. 20, 392-394 (2020). doi:10.1038/s41577-020-0359-5 Medline

15. N. Eroshenko, T. Gill, M. K. Keaveney, G. M. Church, J. M. Trevejo, H. Rajaniemi, Implications of antibody-dependent enhancement of infection for SARS-CoV-2 countermeasures. Nat. Biotechnol. 38, 789-791 (2020). doi:10.1038/s41587020-0577-1 Medline

16. S. Muyldermans, Nanobodies: Natural single-domain antibodies. Annu. Rev. Biochem. 82, 775-797 (2013). doi:10.1146/annurev-biochem-063011-092449 Medline

17. P. Vanlandschoot, C. Stortelers, E. Beirnaert, L. I. Ibañez, B. Schepens, E. Depla, X. Saelens, Nanobodies ${ }^{\circledR}$ : New ammunition to battle viruses. Antiviral Res. 92, 389407 (2011). doi:10.1016/jantiviral.2011.09.002 Medline

18. L. Detalle, T. Stohr, C. Palomo, P. A. Piedra, B. E. Gilbert, V. Mas, A. Millar, U. F. Power, C. Stortelers, K. Allosery, J. A. Melero, E. Depla, Generation and Characterization of ALX-0171, a Potent Novel Therapeutic Nanobody for the Treatment of Respiratory Syncytial Virus Infection. Antimicrob. Agents Chemother. 60, 6-13 (2015). doi:10.1128/AAC.01802-15 Medline

19. R. Konwarh, Nanobodies: Prospects of Expanding the Gamut of Neutralizing Antibodies Against the Novel Coronavirus, SARS-CoV-2. Front. Immunol. 11, 1531 (2020). doi:10.3389/fimmu.2020.01531 Medline

20. T. F. Custódio et al., www.biorxiv.org/content/10.1101/2020.06.23.165415v1 (2020).

21. C. Liu et al., www.biorxiv.org/content/10.1101/2020.03.02.972927v1 (2020).

22. J. Gai et al., www.biorxiv.org/content/10.1101/2020.08.09.242867v1 (2020).

23. C. J. Bracken et al., www.biorxiv.org/content/10.1101/2020.08.08.242511v1 (2020).

24. C. O. Barnes, A. P. West Jr., K. E. Huey-Tubman, M. A. G. Hoffmann, N. G. Sharaf, P. R. Hoffman, N. Koranda, H. B. Gristick, C. Gaebler, F. Muecksch, J. C. C. Lorenzi, S. Finkin, T. Hägglöf, A. Hurley, K. G. Millard, Y. Weisblum, F. Schmidt, T. Hatziioannou, P. D. Bieniasz, M. Caskey, D. F. Robbiani, M. C. Nussenzweig, P. J. 
Bjorkman, Structures of Human Antibodies Bound to SARS-CoV-2 Spike Reveal Common Epitopes and Recurrent Features of Antibodies. Cell 182, 828-842.e16 (2020). doi:10.1016/i.cell.2020.06.025 Medline

25. Y. Xiang et al., www. biorxiv.org/content/10.1101/2020.08.21.261917v1 (2020).

26. W. B. Klimstra, N. L. Tilston-Lunel, S. Nambulli, J. Boslett, C. M. McMillen, T. Gilliland, M. D. Dunn, C. Sun, S. E. Wheeler, A. Wells, A. L. Hartman, A. K. McElroy, D. S. Reed, L. J. Rennick, W. P. Duprex, SARS-CoV-2 growth, furin-cleavage-site adaptation and neutralization using serum from acutely infected hospitalized COVID-19 patients. J. Gen. Virol. (2020). doi:10.1099/jgv.0.001481 Medline

27. M. P. Rout, A. Sali, Principles for Integrative Structural Biology Studies. Cell 177, 1384-1403 (2019). doi:10.1016/j.cell.2019.05.016 Medline

28. C. Yu et al., Charting protein-protein interactions using Cross-Linking Mass Spectrometry (XL-MS). Abstr. Pap. Am. Chem. S. 253 (2017).

29. A. Leitner, M. Faini, F. Stengel, R. Aebersold, Crosslinking and Mass Spectrometry: An Integrated Technology to Understand the Structure and Function of Molecular Machines. Trends Biochem. Sci. 41, 20-32 (2016). doi:10.1016/i.tibs.2015.10.008 Medline

30. B. T. Chait, M. Cadene, P. D. Olinares, M. P. Rout, Y. Shi, Revealing Higher Order Protein Structure Using Mass Spectrometry. J. Am. Soc. Mass Spectrom. 27, $952-$ 965 (2016). doi:10.1007/s13361-016-1385-1 Medline

31. Q. Wang, Y. Zhang, L. Wu, S. Niu, C. Song, Z. Zhang, G. Lu, C. Qiao, Y. Hu, K.-Y. Yuen, Q. Wang, H. Zhou, J. Yan, J. Qi, Structural and Functional Basis of SARSCoV-2 Entry by Using Human ACE2. Cell 181, 894-904.e9 (2020). doi:10.1016/i.cell.2020.03.045 Medline

32. T. Li et al., www.biorxiv.org/content/10.1101/2020.06.09.143438v1 (2020).

33. J. D. Walter et al., www.biorxiv.org/content/10.1101/2020.04.16.045419v1 (2020).

34. J. Huo, A. Le Bas, R. R. Ruza, H. M. E. Duyvesteyn, H. Mikolajek, T. Malinauskas, T. K. Tan, P. Rijal, M. Dumoux, P. N. Ward, J. Ren, D. Zhou, P. J. Harrison, M. Weckener, D. K. Clare, V. K. Vogirala, J. Radecke, L. Moynié, Y. Zhao, J. GilbertJaramillo, M. L. Knight, J. A. Tree, K. R. Buttigieg, N. Coombes, M. J. Elmore, M. W. Carroll, L. Carrique, P. N. M. Shah, W. James, A. R. Townsend, D. I. Stuart, R. J. Owens, J. H. Naismith, Neutralizing nanobodies bind SARS-CoV-2 spike RBD and block interaction with ACE2. Nat. Struct. Mol. Biol. 27, 846-854 (2020). doi:10.1038/s41594-020-0469-6 Medline

35. Y. Shu, J. McCauley, GISAID: Global initiative on sharing all influenza data - from vision to reality. Euro Surveill. 22, 30494 (2017). doi:10.2807/15607917.ES.2017.22.13.30494 Medline

36. A. Baum, B. O. Fulton, E. Wloga, R. Copin, K. E. Pascal, V. Russo, S. Giordano, K. Lanza, N. Negron, M. Ni, Y. Wei, G. S. Atwal, A. J. Murphy, N. Stahl, G. D. Yancopoulos, C. A. Kyratsous, Antibody cocktail to SARS-CoV-2 spike protein prevents rapid mutational escape seen with individual antibodies. Science 369, 1014-1018 (2020). doi:10.1126/science.abd0831 Medline

37. Y. Bar-On, H. Gruell, T. Schoofs, J. A. Pai, L. Nogueira, A. L. Butler, K. Millard, C. Lehmann, I. Suárez, T. Y. Oliveira, T. Karagounis, Y. Z. Cohen, C. Wyen, S. Scholten, L. Handl, S. Belblidia, J. P. Dizon, J. J. Vehreschild, M. Witmer-Pack, I. Shimeliovich, K. Jain, K. Fiddike, K. E. Seaton, N. L. Yates, J. Horowitz, R. M. Gulick, N. Pfeifer, G. D. Tomaras, M. S. Seaman, G. Fätkenheuer, M. Caskey, F. Klein, M. C. Nussenzweig, Safety and antiviral activity of combination HIV-1 broadly neutralizing antibodies in viremic individuals. Nat. Med. 24, 1701-1707 (2018). doi:10.1038/s41591-018-0186-4 Medline

38. M. Marovich, J. R. Mascola, M. S. Cohen, Monoclonal Antibodies for Prevention and Treatment of COVID-19. JAMA 324, 131-132 (2020). doi:10.1001/jama.2020.10245 Medline

39. I. Jovčevska, S. Muyldermans, The Therapeutic Potential of Nanobodies. BioDrugs 34, 11-26 (2020). doi:10.1007/s40259-019-00392-z Medline

40. Z. Shen et al., www.biorxiv.org/content/10.1101/2020.08.19.257725v1 (2020).

41. P. C. Fridy, Y. Li, S. Keegan, M. K. Thompson, I. Nudelman, J. F. Scheid, M. Oeffinger, M. C. Nussenzweig, D. Fenyö, B. T. Chait, M. P. Rout, A robust pipeline for rapid production of versatile nanobody repertoires. Nat. Methods 11, 12531260 (2014). doi:10.1038/nmeth.3170 Medline

42. J. Dunbar, C. M. Deane, ANARCI: Antigen receptor numbering and receptor classification. Bioinformatics 32, 298-300 (2016). Medline

43. S. Kumar, G. Stecher, M. Li, C. Knyaz, K. Tamura, MEGA X: Molecular Evolutionary Genetics Analysis across Computing Platforms. Mol. Biol. Evol. 35, 1547-1549 (2018). doi:10.1093/molbev/msy096 Medline
44. A. Tareen, J. B. Kinney, Logomaker: Beautiful sequence logos in Python. Bioinformatics 36, 2272-2274 (2020). doi:10.1093/bioinformatics/btz921 Medline

45. Y. Shi, J. Fernandez-Martinez, E. Tjioe, R. Pellarin, S. J. Kim, R. Williams, D. Schneidman-Duhovny, A. Sali, M. P. Rout, B. T. Chait, Structural characterization by cross-linking reveals the detailed architecture of a coatomer-related heptameric module from the nuclear pore complex. Mol. Cell. Proteomics 13 , 2927-2943 (2014). doi:10.1074/mcp.M114.041673 Medline

46. Y. Shi, R. Pellarin, P. C. Fridy, J. Fernandez-Martinez, M. K. Thompson, Y. Li, Q. J. Wang, A. Sali, M. P. Rout, B. T. Chait, A strategy for dissecting the architectures of native macromolecular assemblies. Nat. Methods 12, 1135-1138 (2015). doi:10.1038/nmeth.3617 Medline

47. Y. Xiang, Z. Shen, Y. Shi, Chemical Cross-Linking and Mass Spectrometric Analysis of the Endogenous Yeast Exosome Complexes. Methods Mol. Biol. 2062, 383400 (2020). doi:10.1007/978-1-4939-9822-7 18 Medline

48. S. J. Kim, J. Fernandez-Martinez, I. Nudelman, Y. Shi, W. Zhang, B. Raveh, T. Herricks, B. D. Slaughter, J. A. Hogan, P. Upla, I. E. Chemmama, R. Pellarin, I. Echeverria, M. Shivaraju, A. S. Chaudhury, J. Wang, R. Williams, J. R. Unruh, C. H. Greenberg, E. Y. Jacobs, Z. Yu, M. J. de la Cruz, R. Mironska, D. L. Stokes, J. D. Aitchison, M. F. Jarrold, J. L. Gerton, S. J. Ludtke, C. W. Akey, B. T. Chait, A. Sali, M. P. Rout, Integrative structure and functional anatomy of a nuclear pore complex. Nature 555, 475-482 (2018). doi:10.1038/nature26003 Medline

49. A. Šali, T. L. Blundell, Comparative protein modelling by satisfaction of spatial restraints. J. Mol. Biol. 234, 779-815 (1993). doi:10.1006/imbi.1993.1626 Medline

50. A. Fiser, A. Sali, ModLoop: Automated modeling of loops in protein structures. Bioinformatics 19, 2500-2501 (2003). doi:10.1093/bioinformatics/btg362 Medline

51. D. Schneidman-Duhovny, A. Rossi, A. Avila-Sakar, S. J. Kim, J. Velázquez-Muriel, P. Strop, H. Liang, K. A. Krukenberg, M. Liao, H. M. Kim, S. Sobhanifar, V. Dötsch, A. Rajpal, J. Pons, D. A. Agard, Y. Cheng, A. Sali, A method for integrative structure determination of protein-protein complexes. Bioinformatics 28, 3282-3289 (2012). doi:10.1093/bioinformatics/bts628 Medline

52. D. Schneidman-Duhovny, H. J. Wolfson, Modeling of Multimolecular Complexes. Methods Mol. Biol. 2112, 163-174 (2020). doi:10.1007/978-1-0716-0270-6_12 Medline

53. G. Q. Dong, H. Fan, D. Schneidman-Duhovny, B. Webb, A. Sali, Optimized atomic statistical potentials: Assessment of protein interfaces and loops. Bioinformatics 29, 3158-3166 (2013). doi:10.1093/bioinformatics/btt560 Medline

54. Z. Otwinowski, W. Minor, Processing of X-ray diffraction data collected in oscillation mode. Methods Enzymol. 276, 307-326 (1997). doi:10.1016/S00766879(97)76066-X

55. A. J. McCoy, R. W. Grosse-Kunstleve, P. D. Adams, M. D. Winn, L. C. Storoni, R. J. Read, Phaser crystallographic software. J. Appl. Crystallogr. 40, 658-674 (2007). doi:10.1107/S0021889807021206 Medline

56. P. D. Adams, P. V. Afonine, G. Bunkóczi, V. B. Chen, I. W. Davis, N. Echols, J. J. Headd, L.-W. Hung, G. J. Kapral, R. W. Grosse-Kunstleve, A. J. McCoy, N. W. Moriarty, R. Oeffner, R. J. Read, D. C. Richardson, J. S. Richardson, T. C. Terwilliger, P. H. Zwart, PHENIX: A comprehensive Python-based system for macromolecular structure solution. Acta Crystallogr. D 66, 213-221 (2010). doi:10.1107/S0907444909052925 Medline

57. P. Emsley, K. Cowtan, Coot: Model-building tools for molecular graphics. Acta Crystallogr. D 60, 2126-2132 (2004). doi:10.1107/S0907444904019158 Medline

58. C. J. Williams, J. J. Headd, N. W. Moriarty, M. G. Prisant, L. L. Videau, L. N. Deis, V. Verma, D. A. Keedy, B. J. Hintze, V. B. Chen, S. Jain, S. M. Lewis, W. B. Arendall 3rd, J. Snoeyink, P. D. Adams, S. C. Lovell, J. S. Richardson, D. C. Richardson, MolProbity: More and better reference data for improved all-atom structure validation. Protein Sci. 27, 293-315 (2018). doi:10.1002/pro.3330 Medline

59. T. D. Goddard, C. C. Huang, E. C. Meng, E. F. Pettersen, G. S. Couch, J. H. Morris, T. E. Ferrin, UCSF ChimeraX: Meeting modern challenges in visualization and analysis. Protein Sci. 27, 14-25 (2018). doi:10.1002/pro.3235 Medline

60. F. H. Niesen, H. Berglund, M. Vedadi, The use of differential scanning fluorimetry to detect ligand interactions that promote protein stability. Nat. Protoc. 2, 22122221 (2007). doi:10.1038/nprot.2007.321 Medline 


\section{ACKNOWLEDGMENTS}

We thank the staff at the GM/CA of APS in the Argonne National Laboratory (US) for their assistance with X-ray diffraction data collection. We thank the UPMC genome center for Illumina MiSeq, Zhiyi Wei (Southern University of Science and Technology) for the help with crystal structure determination, and Yang Liu for critical reading of the manuscript. Funding: This work was supported by The University of Pittsburgh School of Medicine (Y.S.), a CTSI pilot fund (Y.S.), NIH grant R35GM137905 (Y.S.), The University of Pittsburgh and the Center for Vaccine Research (WPD), NIH grant R35GM128641 (C.Z.), ISF 1466/18 (D.S.), and Israeli Ministry of Science and Technology (D.S.). Author contributions: Y.S. and D.S conceived the study. Y.X. performed most of the experiments. S.N. performed the PRNT SARS-CoV-2 neutralization assay. Z.X. produced the multivalent $\mathrm{Nbs}$ and performed thermostability measurements. C.Z. determined the X-ray structure with the help of H. L.. Y.X., Y.S., D.S., C.Z., Z.S., S.N., and P.D. analyzed the data. Y.S. cheer-led the study and drafted the manuscript. All authors edited the manuscript. Competing interests: Y.X. and Y.S. are coinventors on a provisional patent filed by The University of Pittsburgh covering the Nbs described in this manuscript. Data and materials availability: The coordinates and structure factors for SARS-CoV-2 RBD with Nb20 have been deposited in the Protein Data Bank under the accession codes PDB 7JVB. The proteomics data of chemical crosslink and mass spectrometric analysis (CX-MS) analysis has been deposited into the MassIVE data repository with accession code is MSV000086198. Plasmids are being deposited at Addgene and are available from YS in the interim. This work is licensed under a Creative Commons Attribution 4.0 International (CC BY 4.0) license, which permits unrestricted use, distribution, and reproduction in any medium, provided the original work is properly cited. To view a copy of this license, visit

https://creativecommons.org/licenses/by/4.0/. This license does not apply to figures/photos/artwork or other content included in the article that is credited to a third party; obtain authorization from the rights holder before using such material.

\section{SUPPLEMENTARY MATERIALS}

science.sciencemag.org/cgi/content/full/science.abe4747/DC1

Materials and Methods

Figs. S1 to S13

Tables S1 to S4

References (41-60)

MDAR Reproducibility Checklist

24 August 2020; accepted 28 October 2020

Published online 5 November 2020

10.1126/science.abe4747 

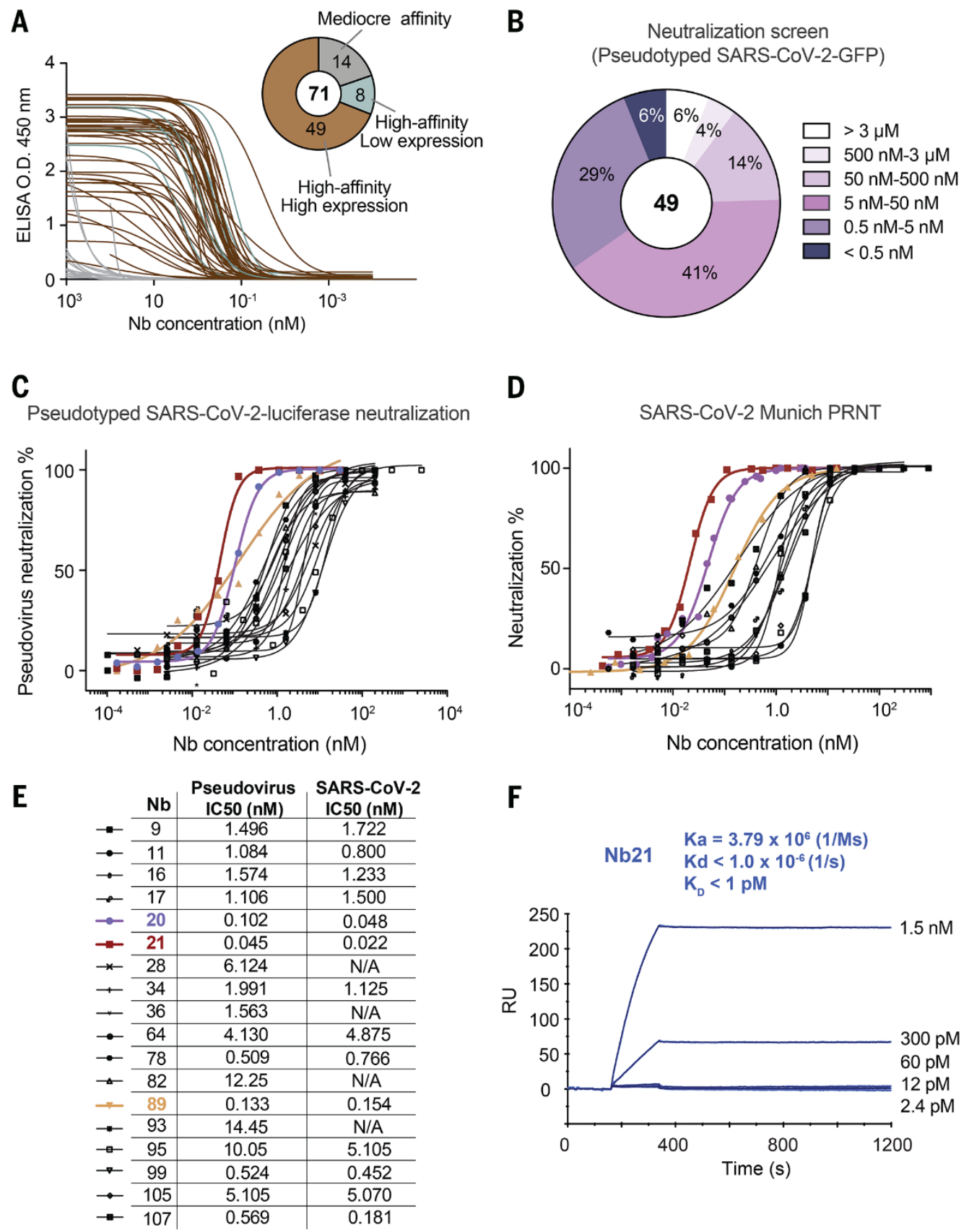

Fig. 1. Production and characterizations of high-affinity RBD Nbs for SARS-CoV-2 neutralization. (A) The binding affinities of $71 \mathrm{Nbs}$ toward RBD by ELISA. The pie chart shows the number of Nbs according to affinity and solubility. (B) Screening of 49 high-affinity $\mathrm{Nbs}$ with high-expression level by SARS-CoV-2-GFP pseudovirus neutralization assay. $\mathrm{n}=1$ for Nbs with neutralization potency $\mathrm{IC} 50<=50 \mathrm{nM}, \mathrm{n}=2$ for Nbs with neutralization potency IC50 > $50 \mathrm{nM}$. (C) The neutralization potency of 18 highly potent Nbs was calculated based on the pseudotyped SARS-CoV-2 neutralization assay (luciferase). Purple, red, and yellow lines denote $\mathrm{Nbs} 20,21$, and 89 with IC50 < $0.2 \mathrm{nM}$. Two different purifications of the pseudovirus were used. The average neutralization percentage was shown for each data point ( $n=5$ for Nbs 20, 21; $n=2$ for all other Nbs). (D) The neutralization potency of 14 neutralizing Nbs by SARS-CoV-2 plaque reduction neutralization test (PRNT). The average neutralization percentage was shown for each data point ( $n=4$ for $\mathrm{Nbs} 20,21$, and 89; $n=2$ for other $\mathrm{Nbs}$ ). (E) A table summary of pseudovirus and SARS-CoV-2 neutralization potencies of $18 \mathrm{Nbs}$. N/A: not tested. (F) The SPR binding kinetics measurement of Nb21. 
A

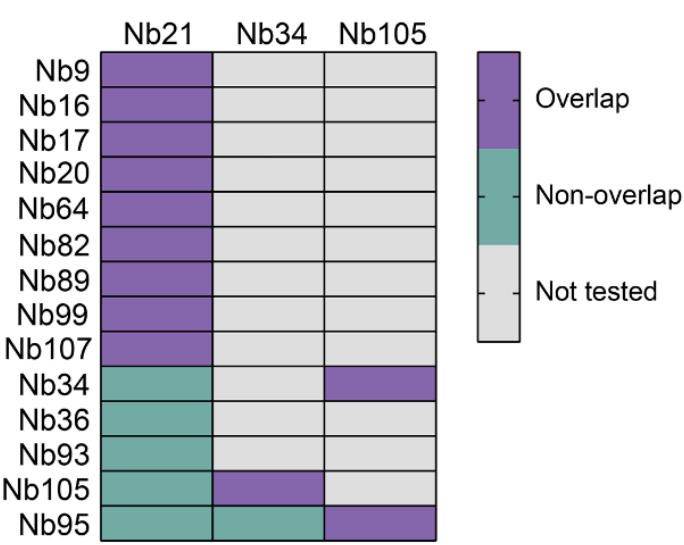

C

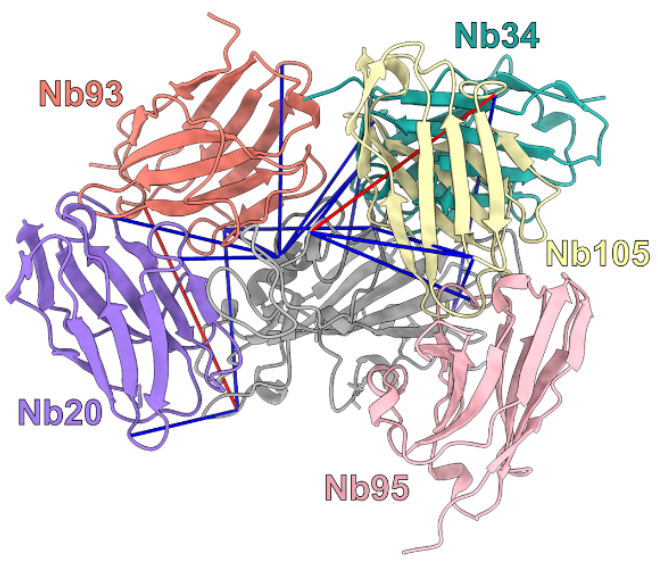

B

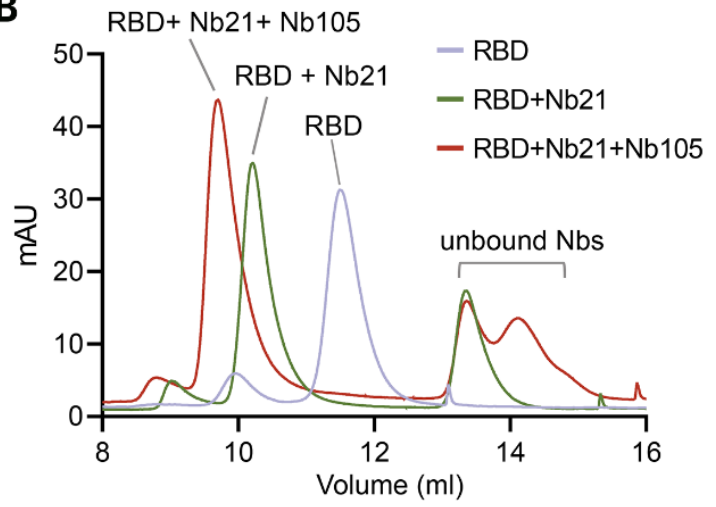

D Epitope IIII

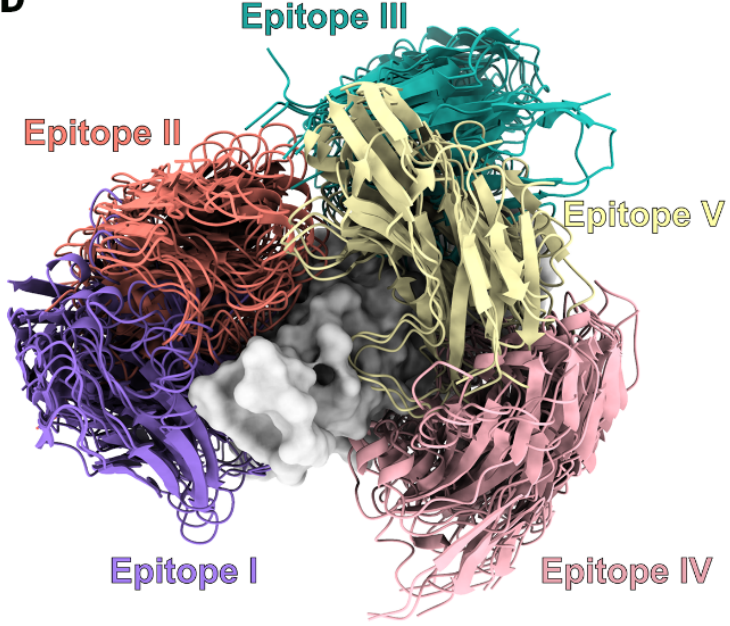

Fig. 2. $\mathrm{Nb}$ epitope mapping by integrative structural proteomics. (A) A summary of $\mathrm{Nb}$ epitopes based on size exclusion chromatography (SEC) analysis. Light salmon color: Nbs that bind the same RBD epitope. Sea green: Nbs of different epitopes. (B) A representation of SEC profiling of RBD, RBD-Nb21 complex, and RBD-Nb21-Nb105 complex. The y-axis represents UV $280 \mathrm{~nm}$ absorbance units (mAu). (C) A cartoon model showing the localization of five Nbs that bind different epitopes: Nb20 (medium purple), Nb34 (light sea green), Nb93 (salmon), Nb105 (pale goldenrod), and Nb95 (light pink) in complex with the RBD (gray). The Blue and red lines represent DSS cross-links shorter or longer than $28 \AA$, respectively. (D) Top 10 scoring cross-linking based models for each $\mathrm{Nb}$ (cartoons) on top of the RBD surface. 
A

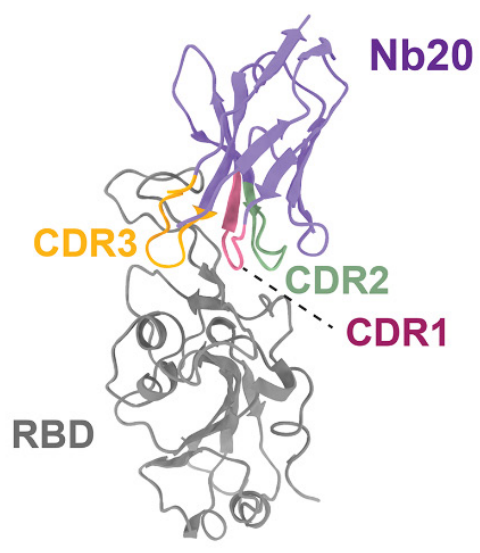

C

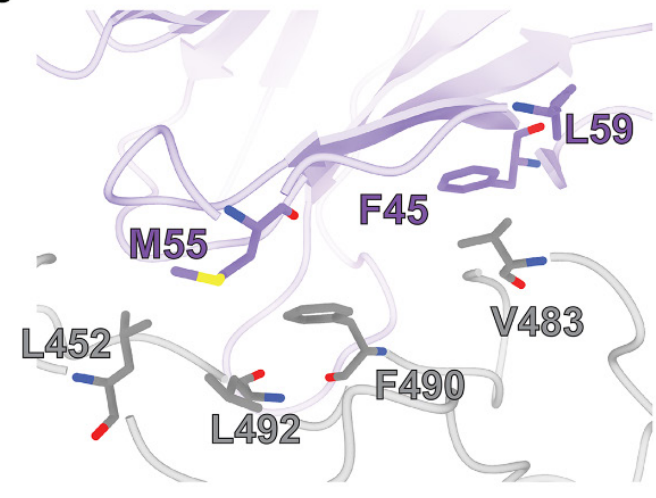

B

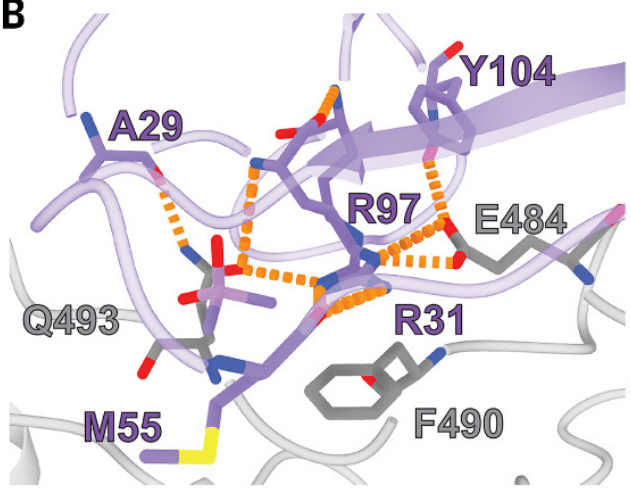

D

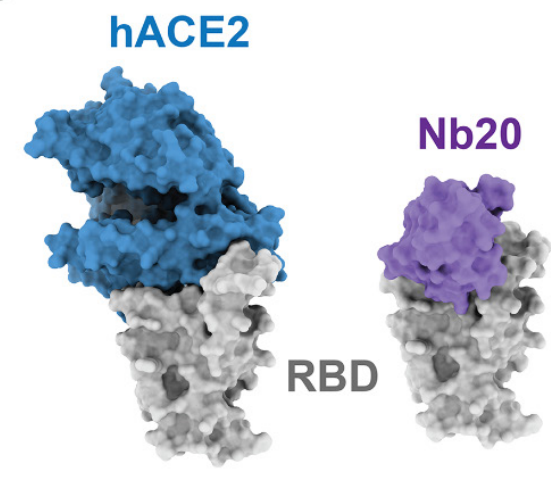

Fig. 3. Crystal structure analysis of an ultrahigh affinity $\mathrm{Nb}$ in complex with the RBD. (A) Cartoon presentation of Nb20 in complex with the RBD. CDR1, 2, and 3 are in red, green, and orange, respectively. (B) Zoomed-in view of an extensive polar interaction network that centers on R35 of Nb20. (C) Zoomed-in view of hydrophobic interactions. (D) Surface presentation of the Nb2O-RBD and hACE2-RBD complex (PDB: 6MOJ). 
A

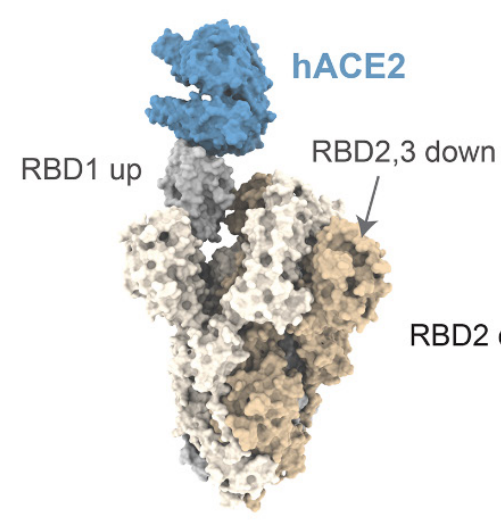

B

Epitope I ( $\mathrm{Nb20/21)}$

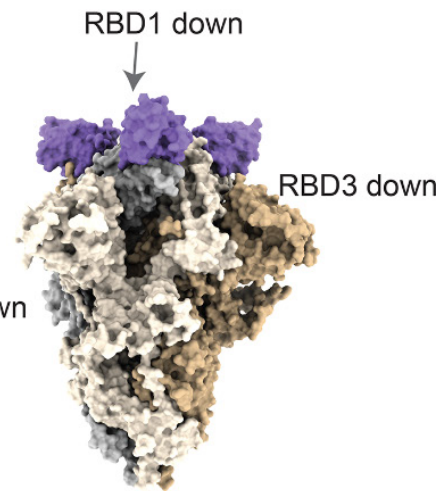

C

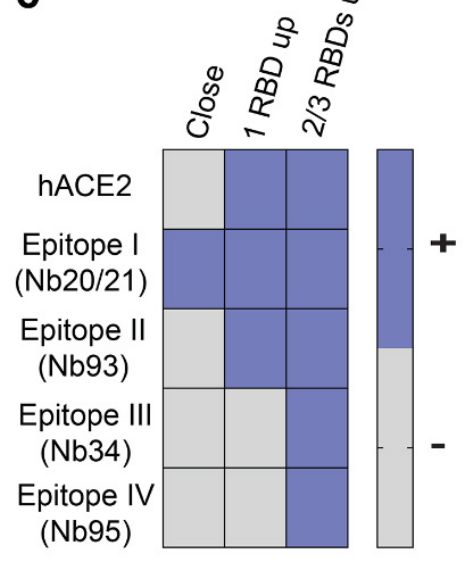

D

Epitope II (Nb93)

RBD1 up

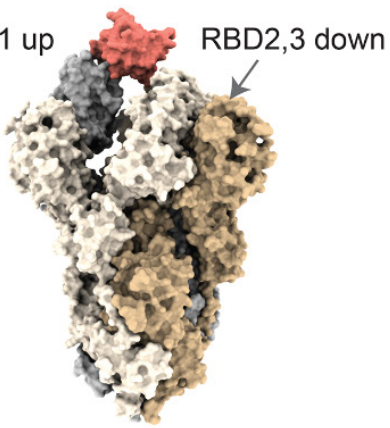

$\mathbf{E}$

Epitope III (Nb34)

RBD3 up

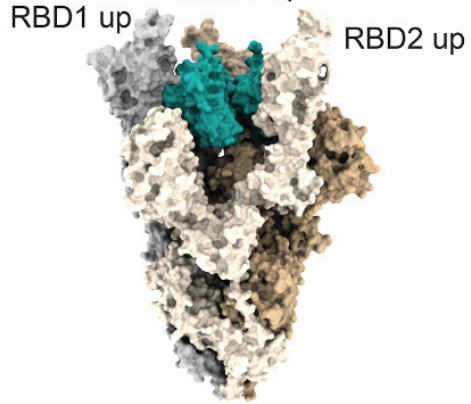

$\mathbf{F}$

Epitope IV (Nb95)

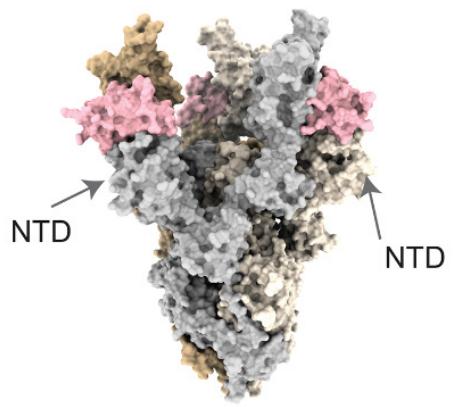

Fig. 4. Potential mechanisms of SARS-CoV-2 neutralization by Nbs. (A) hACE2 (blue) binding to spike trimer conformation (wheat, beige, and gray colors) with one RBD up (PDBs $6 \mathrm{VSB}, 6 \mathrm{LZG}$ ). (B) Nb20 (Epitope I, medium purple) partially overlaps with the hACE2 binding site and can bind the closed spike conformation with all RBDs down (PDB 6VXX). (C) A summary of spike conformations accessible (+) to the Nbs of different epitopes. (D) Nb93 (Epitope II, salmon) partially overlaps with the hACE2 binding site and can bind to spike conformations with at least one RBD up (PDB 6VSB). (E and F) Nb34 (Epitope III, light sea blue) and $\mathrm{Nb95}$ (Epitope IV, light pink) do not overlap with the hACE2 binding site and bind to spike conformations with at least two open RBDs (PDB 6XCN). 
A

Pseudotyped SARS-CoV-2-luciferase neutralization

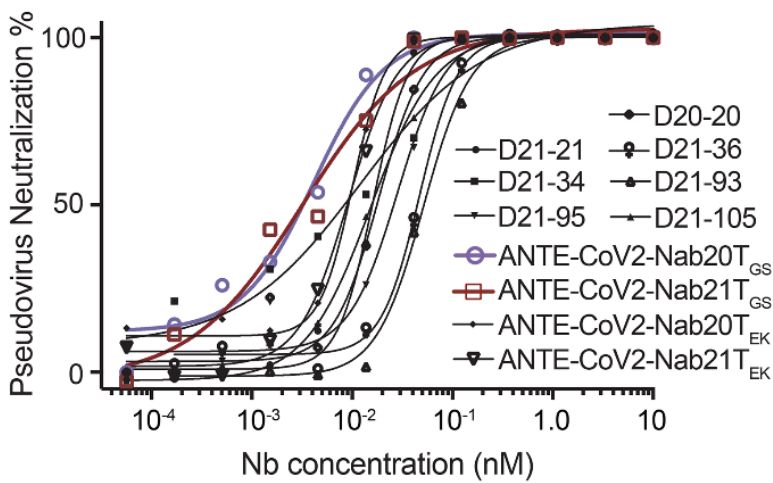

C

\begin{tabular}{|c|c|c|}
\hline Multivalent Nbs & $\begin{array}{l}\text { Pseudovirus } \\
\text { IC50 (pM) }\end{array}$ & $\begin{array}{c}\text { SARS-CoV-2 } \\
\text { IC50 (pM) }\end{array}$ \\
\hline ANTE-CoV2-Nab20Tos & 4.14 & 5.43 \\
\hline ANTE-CoV2-Nab21Tos & 1.32 & 6.04 \\
\hline ANTE-CoV2-Nab20T & 10.09 & 5.75 \\
\hline ANTE-CoV2-Nab21 $\mathrm{T}_{\mathrm{EK}}$ & 9.18 & 9.57 \\
\hline $\begin{array}{c}\mathrm{D} 20-20 \\
\end{array}$ & 18.37 & $\mathrm{~N} / \mathrm{A}$ \\
\hline $\mathrm{D} 21-21$ & 16.11 & $\mathrm{~N} / \mathrm{A}$ \\
\hline D21-34 & 11.99 & $\mathrm{~N} / \mathrm{A}$ \\
\hline $\mathrm{D} 21-36$ & 46.99 & 25.45 \\
\hline D21-93 & 51.40 & N/A \\
\hline D21-95 & 28.31 & $\mathrm{~N} / \mathrm{A}$ \\
\hline D21-105 & 15.35 & $\mathrm{~N} / \mathrm{A}$ \\
\hline
\end{tabular}

B

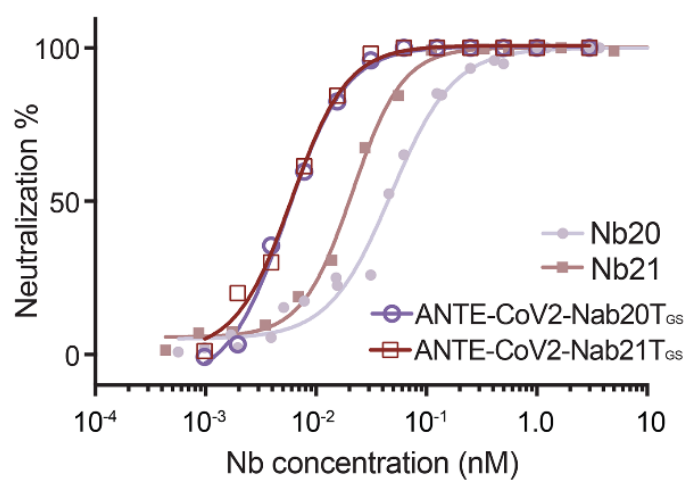

D

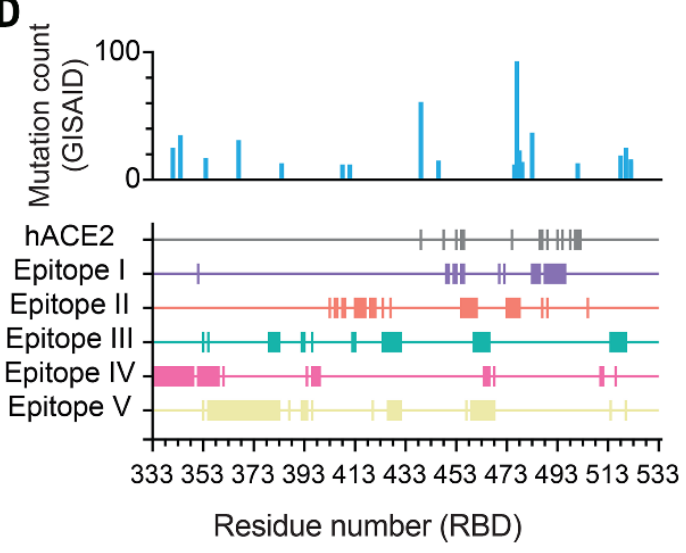

Fig. 5. Development of multivalent $\mathrm{Nb}$ cocktails for highly efficient SARS-CoV-2 neutralization. (A) Pseudotyped SARS-CoV-2 neutralization assay of multivalent Nbs. The average neutralization percentage of each data point was shown $(n=2)$. ANTE-CoV2Nab20TGS/EK: homo-trimeric Nb20 with the GS/EK linker; ANTE-CoV2-Nab21TGS/EK: homo-trimeric Nb21 with the GS/EK linker. (B) SARS-CoV-2 PRNT of monomeric and trimeric forms of Nbs 20 and 21. The average neutralization percentage of each data point was shown ( $n=2$ for the trimers, $n=4$ for the monomers). (C) A summary table of the neutralization potency measurements of the multivalent Nbs. N/A: not tested. (D) Mapping mutations to localization of $\mathrm{Nb}$ epitopes on the RBD. The $\mathrm{x}$-axis corresponds to the RBD residue numbers (333 to 533). Rows in different colors represent different epitope residues. Epitope I: 351, 449-450, 452-453, 455-456, 470, 472, 483-486, 488-496; Epitope II: 403, 405-406, 408,409, 413-417, 419-421, 424, 427, 455-461, 473-478, 487, 489, 505; Epitope III: 53, 355, 379-383, 392-393, 396,412-413, 424-431, 460-466, 514-520; Epitope IV: 333-349, 351-359, 361, 394, 396-399, 464-466, 468, 510-511, 516; Epitope V: 353, 355-383, 387, 392394, 396, 420, 426-431, 457,459-468, 514, 520. 